\title{
Angiosarcoma Following Radiation Therapy for Breast Cancer: Case Presentation and Clinical Management Considerations
}

\author{
Joseph R. Scalea ${ }^{1 *}$, Branko Bojovic ${ }^{2}$, Robin Legros ${ }^{3}$, Julia Choi ${ }^{4}$, Andrea Hebert ${ }^{1}$, Nader Hanna ${ }^{1}$ \\ ${ }^{1}$ Division of General \& Oncologic Surgery, University of Maryland School of Medicine, Baltimore, USA; ${ }^{2}$ Division of Plastic Sur- \\ gery, University of Maryland School of Medicine, Baltimore, USA; ${ }^{3}$ University of Maryland School of Medicine, Baltimore, USA; \\ ${ }^{4}$ Department of Pathology, University of Maryland School of Medicine, Baltimore, USA.

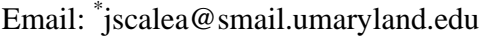

Received May $11^{\text {th }}, 2013$; revised June $11^{\text {th }}, 2013$; accepted June $20^{\text {th }}, 2013$

Copyright (C) 2013 Joseph R. Scalea et al. This is an open access article distributed under the Creative Commons Attribution License, which permits unrestricted use, distribution, and reproduction in any medium, provided the original work is properly cited.

\begin{abstract}
A 59-year-old woman presented with erythema and pruritis of the breast 4.5 years after undergoing lumpectomy and radiation for breast cancer. Biopsy confirmed a diagnosis of angiosarcoma. This tumor stained positive for CD34 as well as $70 \%$ Ki67 prior to therapy initiation. A multidisciplinary approach yielded a plan for neoadjuvant chemoradiation and surgical resection including delayed completion transverse rectus abdominis flap for tissue coverage. Neoadjuvant therapy successfully decreased rates of cellular proliferation, as reflected by a Ki67 of 5\%, at the time of resection. Pathophysiologically, angiosarcomas may be very aggressive and may develop following radiation for breast cancer. Such tumors may become more common as breast cancer therapies, which frequently include radiation therapy, improve with time. Early recognition of angiosarcoma is imperative for successful therapy. These tumors may present with a wide range of symptoms, but may be asymptomatic. Surgical resection is the preferred therapy, but early recognition is critical.
\end{abstract}

Keywords: Angiosarcoma; Breast Cancer; Radiation Therapy; Immunohistochemistry; TRAM Flap

\section{Case Presentation}

Our patient is a very pleasant 59-year-old-female who underwent left breast lumpectomy and axillary lymph node dissection followed by adjuvant chemoradiation in 2006 for a lesion identified as part of her work-up for colon cancer. The patient had appropriate follow-up and underwent routine annual mammographic screening with no signs of recurrence. In April 2011, the patient developed pruritus of the left breast associated with multiple skin lesions. These lesions were described as small, bright red papules that slowly increased in size on an erythematous base. A punch biopsy was performed which showed malignant neoplasm. H\&E staining revealed dermal and subcutaneous tumor with poorly-formed vascular channels (Figure 1(a)). There were atypical epithelioid cells with brisk mitotic activity lining these channels. These findings were consistent with high-grade angiosarcoma. Immunohistochemical staining was positive for p63, vi-

${ }^{*}$ Corresponding author. mentin and CD34 (Figure 1(b)), highlighting the vascularity of the tumor. Stains were negative for AE1/AE3, S100, SMA, desmin, CD68, EMA, CK903, mammaglobin, GCDFP15, FLI1 and HHV8. Staining with Ki67, a protein which is absent during the resting phase of the cell cycle and used as a marker of proliferation, revealed a proliferative index of approximately 70\% (Figure 1(c)). A PET/CT scan was negative for metastasis.

The patient underwent neoadjuvant external beam radiation therapy (XRT). In order to determine the effect of the XRT on the angiosarcoma prior to surgery, immunohistochemistry was re-performed approximately six weeks following XRT. These studies revealed a significant decrease in tumor-associated vascularity, and both CD34 and Ki67 were markedly lower (Figures 2(a)-(c)). Six weeks following XRT, the patient underwent modified radical mastectomy (Figures 3(a)-(e)). Reconstruction was planned as a two-stage procedure, to be performed along with the plastic surgery team. Initially, a split thickness skin-graft (STSG) was used for coverage 


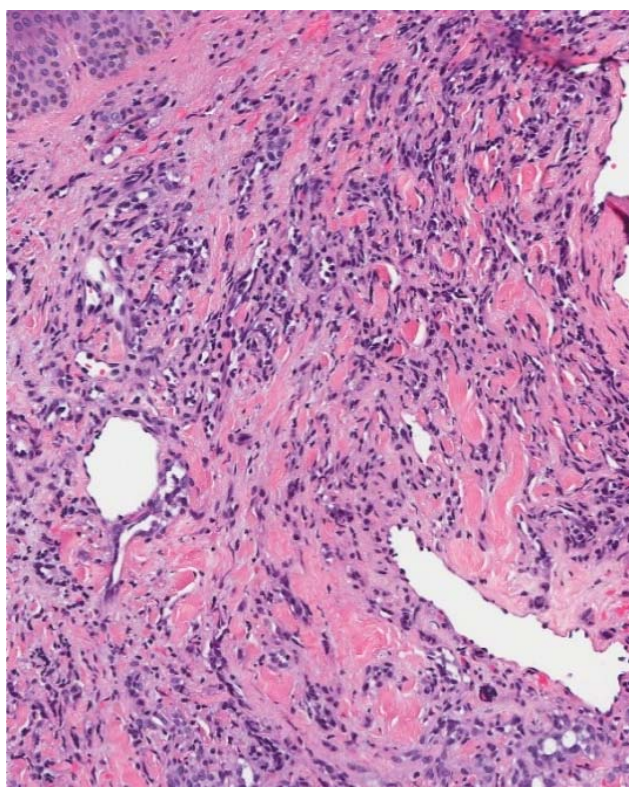

(a)

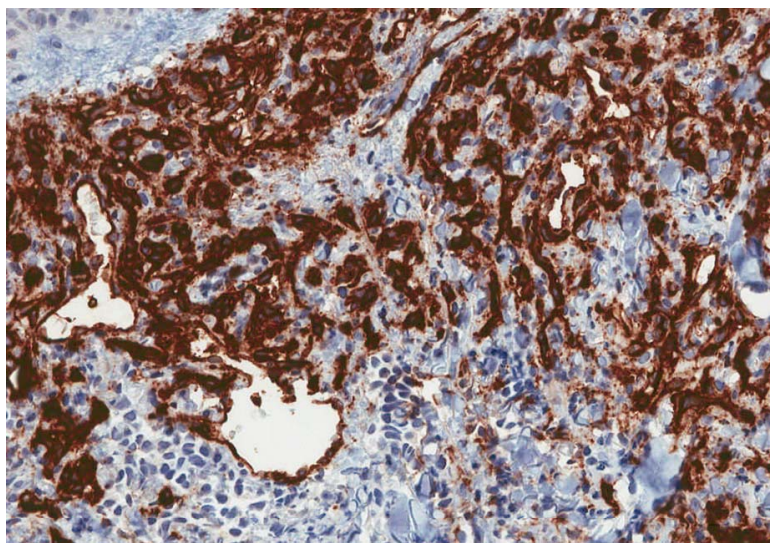

(b)

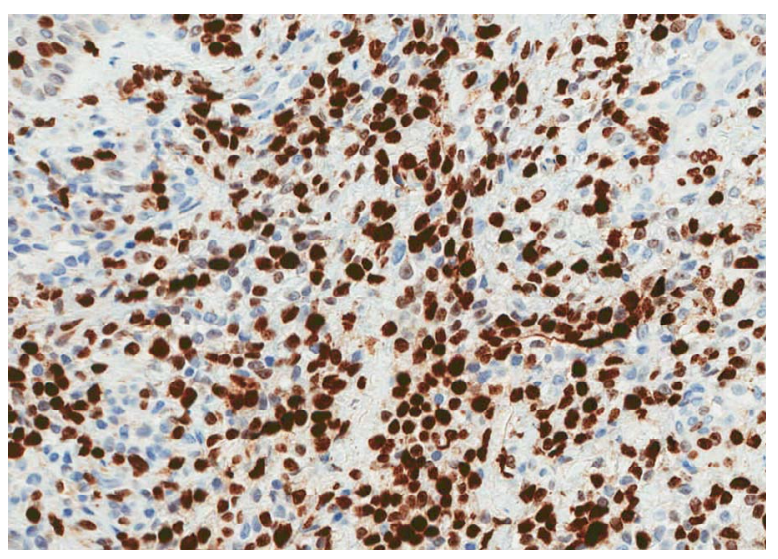

(c)

Figure 1. Pre-radiation biopsy. (a) H\&E stain (mag 100×) revealed high-grade angiosarcoma; (b) Tissue CD34 (mag $200 \times$, dark stain) stains revealed a high-degree of vascularity, consistent with angiosarcoma; (c) Pre-radiation Ki-67 (mag 200×, dark stain) demonstrating a proliferative index of approximately $\mathbf{7 0 \%}$.

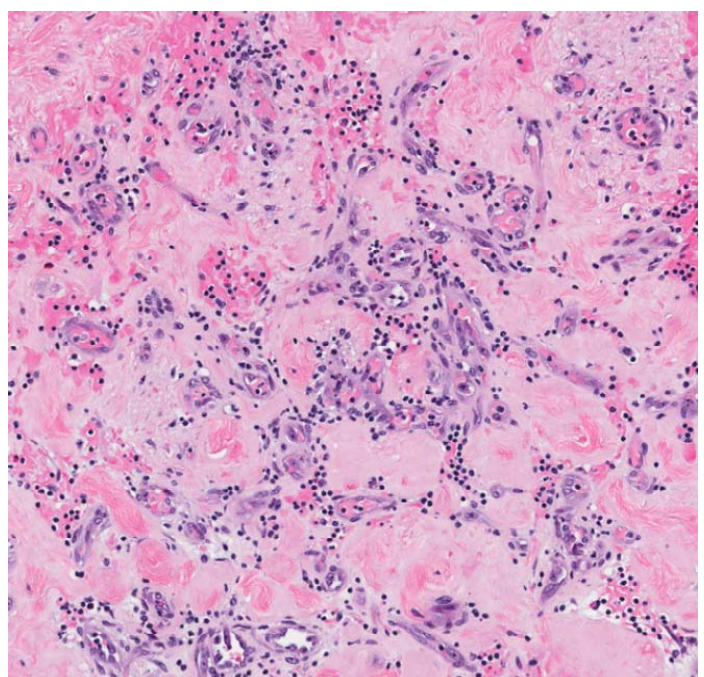

(a)

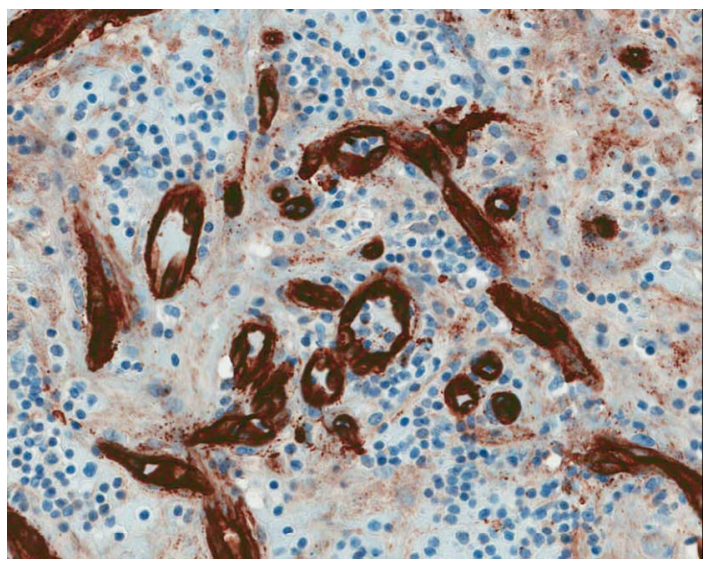

(b)

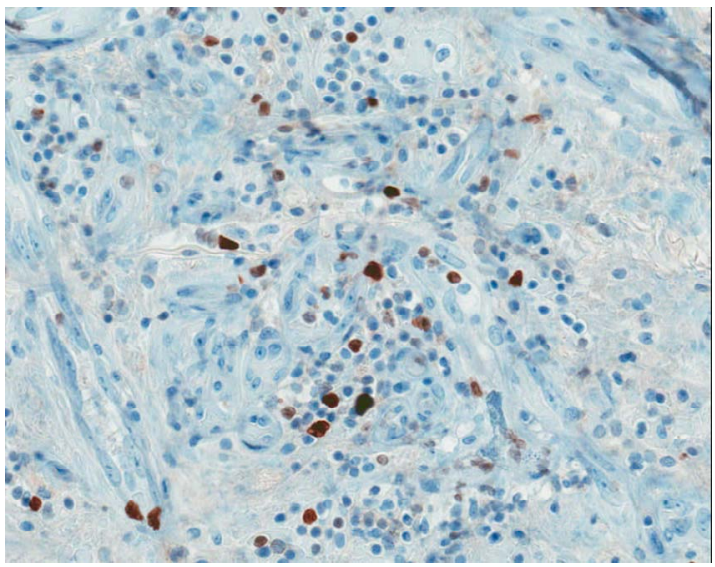

(c)

Figure 2. Response to irradiation. (a) On H\&E (mag 100×) tissue obtained from the mastectomy sample, revealed rare residual microscopic foci of angiosarcoma within the hyalinized and necrotic dermis; (b) Decrease in CD34 (mag $200 \times$, dark stain) concentration following radiation therapy; (c) Post-radiation Ki-67 (mag 200×, dark stain) stain revealing a proliferative index of approximately $5 \%$ at the time of mastectomy. 


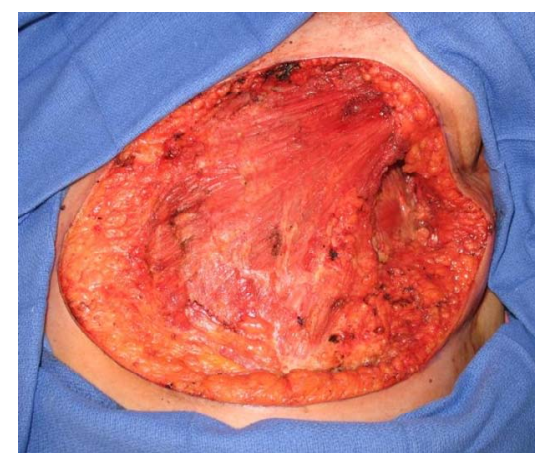

(a)

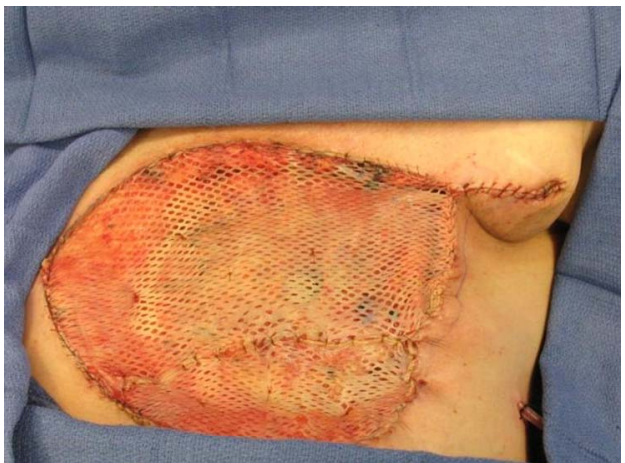

(c)

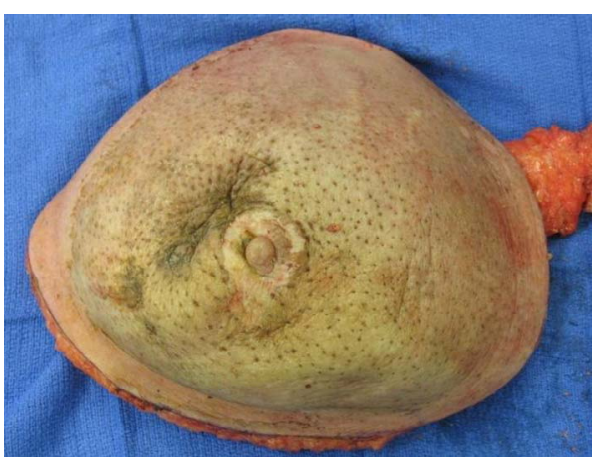

(b)

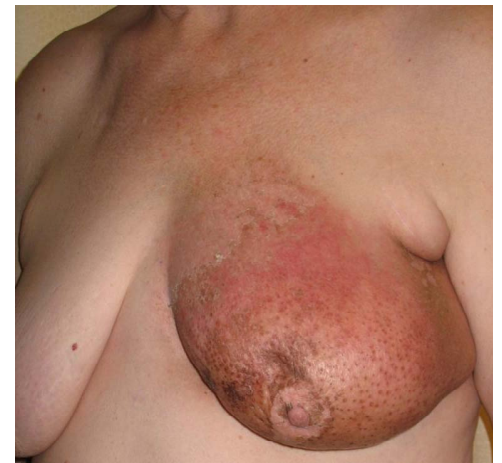

(d)

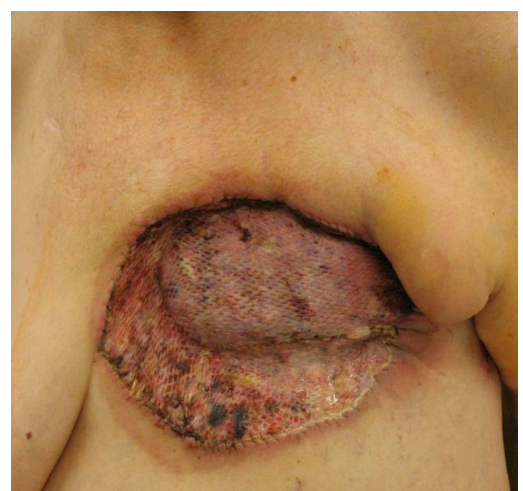

(e)

Figure 3. Surgical management of breast angiosarcoma. (a) Intraoperative photo of post-resection chest wall; (b) Resected breast tissue; (c) STSG coverage of chest wall defect; (d) Pre-resection image; (e) 3 week post-skin graft image.

over the open chest wall wound (Figure 3(b)). This was to be followed by a pedicled transverse rectus abdominis myocutaneous (TRAM) flap for definitive breast reconstruction once pathology confirmed local absence of malignnancy. Post-operative adjuvant chemotherapy had been initiated shortly after her first-stage STSG, as mentioned above.

\section{Disease Process and Epidemiology}

Angiosarcoma (AS) is a heterogenous, aggressive malignancy that arises from vascular endothelium that typically effects elderly white males, however it is a recognized disease process following irradiation for breast cancer in women [1-3]. AS is rare and accounts for less than one percent of soft tissue sarcomas with an estimated annual age-adjusted incidence in the US of 0.21 cases per 100,000 person-years [4]. Primary tumors are broadly categorized as cutaneous and non-cutaneous, with the incidence of each type being roughly equal $[4,5]$. Caucasians are affected more frequently than AfricanAmericans, Asians or Native Americans [2,4,5]. The incidence in men and women increases exponentially after the age of 40 years [4], and according to a publication in the Lancet, the disease presents most commonly in the head and neck (27\%); However, angiosarcoma of the breast, as presented here is the second most common form of angiosarcoma and accounts for $19.7 \%$ of cases. 
In general, the prognosis for angiosarcoma is poor and rigorous pathological investigation should be undertaken to assist in the early diagnosis, treatment, and surveillance of this malignancy [1].

\section{Diagnosis and Treatment}

For therapeutic and epidemiologic purposes, AS can be categorized according to its anatomical site, the natural history of the disease, and its predisposing factors [6]. Cutaneous AS occurs most frequently on the head and neck while lesions on the extremities and trunk are less common [5,6]. The association of cutaneous AS with chronic lymphedema and inflammation, particularly lymphedema following radical mastectomy, is well documented $[3,7]$. These tumors, which appear most commonly on the scalp, typically present as painless, ecchymotic or nodular lesions with occasional bleeding or ulceration $[3,6,7]$. Their nondescript clinical features often lead to misdiagnosis of these tumors as benign disease [3,7]. AS associated with lymphedematous extremities (Stewart-Treves syndrome) presents with similar features and typically presents years after the development of lymphedema [6,8]. Regular follow-up of these patients with gadolinium-enhanced MRI may be indicated to increase detection of AS [7].

Critical to the diagnosis of angiosarcoma are the immunohistochemical stains. Pathologically, these tumors typically express endothelial markers such as von Willebrand factor, CD34, CD31, and vascular endothelial growth factor (VEGF) [1]. As was shown for the patient in this study, markers, such as CD34 can be helpful in monitoring the success of cancer therapies. However, it should be noted, that with de-differentiation, tumors may lead to loss of cellular markers [1]. Here, the tumor was positive for p63, which may alter VEGF expression that may be seen in vascular neoplasia. Vimentin was also positive on initial staining and is helpful in confirming the diagnosis of an epithelioid angiosarcoma, in the setting of CD31 and/or CD34 positivity [9]. It is now appreciated that atypical vascular lesions (AVLs) are a precursors to AS. These two diseases (AVLs and AS) likely exist on a spectrum [10,11]. AVLs typically begin as benign-appearing, well-circumscribed collections of cutaneous vessels [12]. There also appears to be a clear link between radiation exposure and the development to AVLs [10]. Retrospective data has shown that, women who develop AS are typically older and experience a shorter duration between radiation exposure and the diagnosis of dermatologic pathology [10]. In a recent report of 11 patients with radiation associated AVLs, some whom subsequently developed AS, authors observed that AVLs occurred at a mean of 4.3 years following radiation, and that AS occurred slightly later (mean $=5.0$ years). In addition, whereas staining for CD34 was similar among patients with AVL and AS (as was observed with our patient), investigators found that VEGFR-3 expression was greater in those patients with AVLs that went on to develop AS [10].

AS of the breast may arise spontaneously or be associated with prior radiation therapy. In another series of 57 patients with breast AS, 27 patients (47\%) had previously received breast irradiation; only three of these patients experienced upper extremity lymphedema [6]. The development of AS in previously irradiated fields such as the face, tongue, pleura and GI tract has been reported in other retrospective publications [2].

Primary AS of deep, soft tissue and of solid organs may be asymptomatic [6,7]. In symptomatic patients, the clinical presentation of AS is related to tissue origin. AS of the spleen and liver may present as a painful mass whereas cardiac tumors may lead to pericardial effusion [5]. Both primary and metastatic AS may be found in virtually any deep soft tissue, organ or bone [2,13]. At diagnosis, these tumors are frequently high-grade, poorly differentiated neoplasms, making complete surgical resection difficult and both local and distal recurrence common [2,3,7]. Rare cases of AS associated with foreign material have been reported $[13,14]$.

\section{Outcomes}

Because breast conservation therapy typically involves lumpectomy and radiation, and because many more women are electing to have breast conservation therapy, the incidence of angiosarcoma is expected to increase [15]. However, some authors have reported lower-than expected rates of angiosarcoma secondary to radiation exposure [15]. In 2008, a retrospective review of 32 cases of AVLs in women that had undergone breast conservation therapy brought more attention the incidence of this disease process [12].

Our patient, as the data above describes, underwent lumpectomy and radiation. After development of her angiosarcoma, a careful clinico-pathologic analysis was performed to clarify her tumor type. In doing so, CD34 and Ki67 (Figures 1 and 2) were followed by biopsy, in order to determine the clinical success of radiation therapy. We demonstrated successful decrease in these markers (CD34, Ki67, etc.) indicating decreased rate of tumor growth following XRT, as has been shown for other tumor markers such as VEGF [16].

Five-year survival in AS patients in approximately $45 \%$ based on population surveillance data [5]. Surgical resection is the standard of care for AS, and patients with resectable disease experience increased survival compared to patients with unresectable tumors [13]. In one large series, overall survival (OS) was $48 \%$ in patients 
with localized disease and $23 \%$ in patients with metastatic disease $(p<0.001)$ [6]. Univariate analysis in a separate series revealed a statistically significant difference $(p<0.001)$ in disease-specific survival (DSS) between patients with advanced, unresectable AS at cutaneous (median DSS $=10.3 \mathrm{mo}$ ) and deep soft tissue sites (median DSS = $2.8 \mathrm{mo}$ ) [7]. For patients with localized or advanced disease, another series demonstrated improved overall survival for patients with superficial (3.6 $\pm 1.0 \mathrm{yrs}$ ) versus deep ( $2.3 \pm 0.5$ yrs) tumors [2]. In patients with localized cutaneous disease, primary tumor origin in the head/neck, extremities or breast was associated with significantly longer survival than tumors arising in the trunk (median survival: $3.6 \pm 0.5$ yrs vs. $2.1 \pm$ 0.2 yrs; $p=0.01$ ) [2]. Among patients with primary breast AS, the presence of preexisting lymphedema and radiation fields were negative prognostic indicators for local and distal disease recurrence and overall survival $[2,7]$. Several studies have failed to find a significant association between microscopically positive surgical margins and poor disease outcomes [2,13]. Furthermore, the role of adjuvant and neoadjuvant chemoradiation for patients with resectable AS is unclear. In our patient, we observed favorable histologic results using neoadjuvant irradiation. In two case series, adjuvant radiotherapy provided no survival benefit in AS patients irrespective of tumor margin status [2,6]. Trials of adjuvant chemotherapy using paclitaxel-based regimens have not shown a survival advantage over more widely used doxorubicinbased therapies $[2,6]$. Paclitaxel may, however, may be of greater benefit than doxorubicin in patients with advanced, unresectable AS [13].

\section{Recommendations}

Angiosarcoma is incompletely understood and is generally very aggressive. Unfortunately, AS is likely to increase in incidence. With the appropriate pathological analyses it is possible 1) to make an early diagnosis, 2) to monitor the tumor for treatment efficacy, and 3) to survey for recurrence. Interestingly our patient's malignnancy was positive for p63, which alters VEGF expression [17] and is identified in approximately $22 \%$ of angiosarcomas. However, this marker is somewhat nonspecific and its presence should be carefully interpreted. In contrast, the identification of established markers such as CD34 may yield more helpful prognostic and surveillance information [18]. Based on clinical and pathologic characteristics, we used a staged, multidisciplinary approach to post-mastectomy reconstruction, which favors definitive reconstruction only after appropriate time for adjuvant treatment and follow-up to rule-out disease recurrence has occurred. Unfortunately, our patient did have local recurrence following the first-stage of treatment.

\section{REFERENCES}

[1] R. J. Young, N. J. Brown, M. W. Reed, D. Hughes and P. J. Woll, “Angiosarcoma,” Lancet Oncology, Vol. 11, No. 10, 2010, pp. 983-991. http://dx.doi.org/10.1016/S1470-2045(10)70023-1

[2] M. G. Fury, C. R. Antonescu, K. J. Van Zee, M. F. Brennan and R. G. Maki, "A 14-Year Retrospective Review of Angiosarcoma: Clinical Characteristics, Prognostic Factors, and Treatment Outcomes with Surgery and Chemotherapy,” Cancer Journal, Vol. 11, No. 3, 2005, pp. 241-247. http://dx.doi.org/10.1097/00130404-200505000-00011

[3] C. Leowardi, U. Hinz, Y. Hormann, M. N. Wente, G. Mechtersheimer, F. Willeke, et al., "Malignant Vascular Tumors: Clinical Presentation, Surgical Therapy, and LongTerm Prognosis,” Annals of Surgical Oncology, Vol. 12, No. 12, 2005, pp. 1090-1101. http://dx.doi.org/10.1245/ASO.2005.09.002

[4] J. R. Toro, L. B. Travis, H. J. Wu, K. Zhu, C. D. M. Fletcher and S. S. Devesa, "Incidence Patterns of Soft Tissue Sarcomas, Regardless of Primary Site, in the Surveillance, Epidemiology, and End Results Program, 19782001: An Analysis of 26,758 Cases,” International Journal of Cancer, Vol. 119, No. 12, 2006, pp. 2922-2930. http://dx.doi.org/10.1002/ijc.22239

[5] P. Rouhani, D. M. C. Fletcher, S. S. Devesa and J. R. Toro, "Cutaneous Soft Tissue Sarcoma Incidence Patterns in the U.S.: An Analysis of 12,144 Cases," Cancer, Vol. 113, No. 3, 2008, pp. 616-627. http://dx.doi.org/10.1002/cncr.23571

[6] J. Fayette, E. Martin, S. Piperno-Neumann, A. Le Cesne, C. Robert, S. Bonvalot, et al., "Angiosarcomas, a Heterogenous Group of Sarcomas with Specific Behaviors Depending on Primary Site: A Retrospective Study of 161 Cases,” Annals of Oncology, Vol. 18, No. 12, 2007, pp. 2030-2036. http://dx.doi.org/10.1093/annonc/mdm381

[7] J. A. Abraham, F. J. Hornicek, A. M. Kaufman, D. C. Harmon, D. S. Springfield, K. A. Raskin, et al., "Treatment and Outcome of 82 Patients with Angiosarcoma," Annals of Surgical Oncology, Vol. 14, No. 6, 2007, pp. 1953-1967. http://dx.doi.org/10.1245/s10434-006-9335-y

[8] S. T. Schindera, M. Streit, U. Kaelin, E. Stauffer, L. Steinbach and S. E. Anderson, "Stewart-Treves Syndrome: MR Imaging of a Postmastectomy Upper-Limb Chronic Lymphedema with Angiosarcoma," Skeletal Radiology, Vol. 34, No. 3, 2005, pp. 156-160. http://dx.doi.org/10.1007/s00256-004-0807-5

[9] A. Abu-Zaid and S. Mohammed, "Primary Pleural Angiosarcoma in a 63-Year-Old Gentleman,” Case Reports in Pulmonology, Vol. 2013, 2013, Article ID: 974567.

[10] I. W. Mattoch, J. B. Robbins, R. L. Kempson and S. Kohler, "Post-Radiotherapy Vascular Proliferations in Mammary Skin: A Clinicopathologic Study of 11 Cases," Journal of the American Academy of Dermatology, Vol. 57, No. 1, 2007, pp. 126-133. http://dx.doi.org/10.1016/j.jaad.2006.10.025

[11] C. Gengler, J. M. Coindre, A. Leroux, M. Trassard, D. Ranchère-Vince, I. Valo, J. J. Michels and L. Guillou, 
"Vascular Proliferations of the Skin after Radiation Therapy for Breast Cancer: Clinicopathologic Analysis of a Series in Favor of a Benign Process: A Study from the French Sarcoma Group,” Cancer, Vol. 109, No. 8, 2007, pp. 1584-1598. http://dx.doi.org/10.1002/cncr.22586

[12] K. T. Patton, A. T. Deyrup and S. W. Weiss, "Atypical Vascular Lesions after Surgery and Radiation of the Breast: A Clinicopathologic Study of 32 Cases Analyzing Histologic Heterogeneity and Association with Angiosarcoma,” American Journal of Surgical Pathology, Vol. 32, No. 6, 2008, pp. 943-950. http://dx.doi.org/10.1097/PAS.0b013e31815bf8fe

[13] G. Lahat, A. R. Dhuka, H. Hallevi, L. Xiao, C. Zou, K. D. Smith, et al., "Angiosarcoma: Clinical and Molecular Insights," Annals of Surgery, Vol. 251, No. 6, 2010, pp. 1098-1106. http://dx.doi.org/10.1097/SLA.0b013e3181dbb75a

[14] Y. T. Joo, C. Y. Jeong, E. J. Jung, Y. J. Lee, S. C. Hong, S. K. Choi, et al., "Intra-Abdominal Angiosarcoma Developing in a Capsule of a Foreign Body: Report of a Case with Associated Hemorrhagic Diathesis," World Journal of Surgical Oncology, Vol. 3, 2005, p. 60. http://dx.doi.org/10.1186/1477-7819-3-60

[15] S. Fineberg and P. P. Rosen, "Cutaneous Angiosarcoma and Atypical Vascular Lesions of the Skin and Breast af- ter Radiation Therapy for Breast Carcinoma,” American Journal of Clinical Pathology, Vol. 102, No. 6, 1994, pp. 757-763.

[16] Y. Toiyama, Y. Inoue, S. Saigusa, Y. Okugawa, T. Yokoe, K. Tanaka, C. Miki and M. Kusunoki, "Gene Expression Profiles of Epidermal Growth Factor Receptor, Vascular Endothelial Growth Factor and Hypoxia-Inducible Factor-1 with Special Reference to Local Responsiveness to Neoadjuvant Chemoradiotherapy and Disease Recurrence after Rectal Cancer Surgery,” Clinical Oncology (Royal College of Radiologists), Vol. 22, No. 4, 2010, pp. 272280. http://dx.doi.org/10.1016/j.clon.2010.01.001

[17] M. Senoo, Y. Matsumura and S. Habu, “TAp63gamma (p51A) and dNp63alpha (p73L), Two Major Isoforms of the p63 Gene, Exert Opposite Effects on the Vascular Endothelial Growth Factor (VEGF) Gene Expression,” Oncogene, Vol. 21, No. 16, 2002, pp. 2455-2465. http://dx.doi.org/10.1038/sj.onc.1205330

[18] M. E. Kallen, F. G. Nunes Rosado, A. L. Gonzalez, M. E. Sanders and J. M. Cates, "Occasional Staining for p63 in Malignant Vascular Tumors: A Potential Diagnostic Pitfall,” Pathology \& Oncology Research, Vol. 18, No. 1, 2012, pp. 97-100.

http://dx.doi.org/10.1007/s12253-011-9426-3 